\title{
Managing Audio Monitoring Data with SIMON - Concept for Data Administration, Online Repository and Dissemination
}

\author{
Christian Köhler $\ddagger$ \\ $\ddagger$ Ganzgraph, Bonn, Germany
}

Corresponding author: Christian Köhler (koehler@ganzgraph.de)

Received: 29 Sep 2020 | Published: 30 Sep 2020

Citation: Köhler C (2020) Managing Audio Monitoring Data with SIMON - Concept for Data Administration, Online Repository and Dissemination. Biodiversity Information Science and Standards 4: e59108. https://doi.org/10.3897/biss.4.59108

\begin{abstract}
Automated observations of natural occurrences play a key role in monitoring biodiversity worldwide. With the development of affordable hardware like the AudioMoth (Hill et al. 2019) acoustic logger, large scale and long-term monitoring has come within reach. However, data management and dissemination of monitoring data remain challenging, as the development of software and the infrastructure for the management of monitoring data lag behind. We want to fill this gap, providing a complete audio monitoring solution from affordable audio monitoring hardware, custom data management tools and storage infrastructure based on open source hard- and software, biodiversity information standards and integrable interfaces.
\end{abstract}

The Scientific Monitoring Data Management and Online Repository (SIMON) consists of a portable data collector and a connected online repository. The data collector, a device for the automated extraction of the audio data from the audio loggers in the field, stores the data and metadata in an internal cache. Once connected to the internet via WiFi or a cable connection, the data are automatically uploaded to an online repository for automated analysis, annotation, data management and dissemination.

To prevent SIMON from becoming yet another proprietary storage, the FAIR principles (F indable, Accessible, Interoperable, and Re-usable) Wilkinson et al. (2016) are at the very 
core of data managed in the online repository. We plan to offer an API (application programming interface) to disseminate data to established data infrastructures. A second API will allow the use of external services for data enrichment. While in the planning phase, we would like to take the opportunity to discuss with domain experts the requirements and implementation of different standards-namely ABCD (Access to Biological Collections Data task group, Biodiversity Information Standards (TDWG) 2007), Darwin Core (Darwin Core Task Group, Biodiversity Information Standards (TDWG) 2009) and Darwin Core Archive (Remsen et al. 2017)—connecting to external services and targeting data infrastructures.

\section{Keywords}

open source software, FAIR, biodiversity information standards

\section{Presenting author}

Christian Köhler

\section{Hosting institution}

ganzgraph, Bonn, Germany

\section{References}

- Access to Biological Collections Data task group, Biodiversity Information Standards (TDWG) (2007) Access to Biological Collection Data (ABCD) Schema. http:// www.tdwg.org/standards/115. Accessed on: 2020-7-09.

- Darwin Core Task Group, Biodiversity Information Standards (TDWG) (2009) Darwin Core. http://www.tdwg.org/standards/450. Accessed on: 2020-7-09.

- Hill A, Prince P, Snaddon J, Doncaster CP, Rogers A (2019) AudioMoth: A low-cost acoustic device for monitoring biodiversity and the environment. HardwareX 6 https:// doi.org/10.1016/j.ohx.2019.e00073

- $\quad$ Remsen D, Braak K, Döring M, Robertson T (2017) Darwin Core Archives - How-to Guide, version 2.0. https://github.com/gbif/ipt/wiki/DwCAHowToGuide. Accessed on: 2020-7-09.

- Wilkinson M, Dumontier M, Aalbersberg IJ, Appleton G, Axton M, Baak A, Blomberg N, Boiten J, da Silva Santos LB, Bourne P, Bouwman J, Brookes A, Clark T, Crosas M, Dillo I, Dumon O, Edmunds S, Evelo C, Finkers R, Gonzalez-Beltran A, Gray AG, Groth P, Goble C, Grethe J, Heringa J, 't Hoen PC, Hooft R, Kuhn T, Kok R, Kok J, Lusher S, Martone M, Mons A, Packer A, Persson B, Rocca-Serra P, Roos M, van Schaik R, Sansone S, Schultes E, Sengstag T, Slater T, Strawn G, Swertz M, Thompson M, van der Lei J, van Mulligen E, Velterop J, Waagmeester A, Wittenburg P, Wolstencroft K, 
Zhao J, Mons B (2016) The FAIR Guiding Principles for scientific data management and stewardship. Scientific Data 3 (1). https://doi.org/10.1038/sdata.2016.18 